\title{
Deprivation of arginine by recombinant human arginase in prostate cancer cells
}

\author{
Eddy C Hsueh ${ }^{1}$, Stephanie M Knebel', Wai-Hung Lo², Yun-Chung Leung ${ }^{2}$, Paul Ning-Man Cheng ${ }^{3}$ and \\ Chung-Tsen Hsueh ${ }^{*}$
}

\begin{abstract}
Background: Recombinant human arginase (rhArg) has been developed for arginine deprivation therapy in cancer, and is currently under clinical investigation. During pre-clinical evaluation, rhArg has exhibited significant antiproliferative activity in cancer cells deficient in the expression of ornithine carbamoyl transferase (OCT). Interestingly, a variety of cancer cells such as melanoma and prostate cancer deficient in argininosuccinate synthetase (ASS) are sensitive to arginine deprivation by arginine deiminase. In this study, we investigated levels of gene expression of $\mathrm{OCT}$ and ASS, and the effects of rhArg in human prostate cancer cells: LNCaP (androgen-dependent), PC-3 and DU145 (both androgen-independent).

Results: Quantitative real-time PCR showed minimal to absent gene expression of $\mathrm{OCT}$, but ample expression of ASS expression in all 3 cell lines. Cell viability assay after 72-h exposure of rhArg showed all 3 lines had half maximal inhibitory concentration less than or equal to $0.02 \mathrm{U} / \mathrm{ml}$. Addition of ornithine to cell culture media failed to rescue these cells from rhArg-mediated cytotoxicity.

Decreased phosphorylation of 4E-BP1, a downstream effector of mammalian target of rapamycin (mTOR), was noted in DU-145 and PC-3 after exposure to rhArg. Moreover, there was no significant apoptosis induction after arginine deprivation by rhArg in all 3 prostate cancer cell lines.

Conclusion: rhArg causes significant cytotoxicity in LNCaP, DU-145 and PC-3 prostate cancer cells which all demonstrate decreased OCT expression. Inhibition of mTOR manifested by hypophosphorylation of 4E-BP1 suggests autophagy is involved as alternative cell death mechanism. rhArg demonstrates a promising novel agent for prostate cancer treatment.
\end{abstract}

Keywords: Arginase, Prostate cancer, Ornithine carbamoyl transferase, Argininosuccinate synthetase, Mammalian target of rapamycin, Autophagy

\section{Background}

Arginine, a nonessential amino acid, is involved in many biochemical processes besides protein synthesis, such as urea cycle and biosyntheses of creatine, polyamine and nitric oxide [1]. In human tissue, arginine is obtained via protein degradation and dietary intake. Additionally, normal cells can synthesize arginine intracellularly from ornithine, mediated by ornithine carbamoyl transferase (OCT) which metabolizes ornithine and carbamoly phosphate into citrulline; argininosuccinate synthetase (ASS) and argininosuccinate lyase subsequently convert citrulline to arginine [2].

\footnotetext{
* Correspondence: chsueh@llu.edu

${ }^{4}$ Division of Medical Oncology and Hematology, Loma Linda University, Loma Linda, CA92354, USA

Full list of author information is available at the end of the article
}

OCT is highly expressed in liver and small intestine, and catabolizes the conversion of ornithine to citrulline [3]. However, OCT expression in cancer and other normal tissues is mostly down-regulated due to epigenetic changes such as DNA hypermethylation [4].

For years, depletion of arginine has been shown to be an effective and promising anti-cancer treatment in vitro and in vivo $[5,6]$. By culturing cells in the media depleted of arginine, a variety of human cancer cells have been found to be auxotrophic for arginine, depletion of which results in cell death [7-9]. Further studies have indicated deficiencies in either ASS or OCT expression contributes to arginine auxotrophy in melanoma and hepatocellular carcinoma [10-13]. Arginine can be degraded by three enzymes: arginase, arginine decarboxylase and arginine deiminase (ADI). Both

\section{Biomed Central}


arginine decarboxylase and ADI are not expressed in mammalian cells [2,14]. ADI, an enzyme isolated from $\mathrm{Myco-}$ plasma, catabolizes arginine to citrulline and ammonia. Pegylated ADI (ADI-PEG20), significantly reduces antigenicity of ADI, and has been evaluated clinically in patients with advanced hepatocellular carcinoma and melanoma $[15,16]$. The sensitivity of ADI-PEG20 in cancer seems to correspond with deficient expression of ASS. Resistance to ADI-PEG20 has been identified in hepatocellular carcinoma, melanoma and prostate cancer cells expressing ASS $[10,17,18]$.

Arginase participates in the urea cycle, and catabolizes arginine to ornithine and urea [19]. Recombinant human arginase (rhArg) has been developed for arginine deprivation therapy in cancer, and demonstrated significant cytotoxicity in hepatocellular carcinoma and melanoma, in vitro and in vivo [11-13]. In the setting of OCT deficiency, rhArg eliminates extracellular arginine and results in depletion of intracellular arginine; however in cells expressing OCT, intermediate metabolite such as ornithine can be converted to arginine to avoid intracellular depletion of arginine [20]. It has been demonstrated that OCT deficiency in hepatocellular cancer and melanoma contributes to their sensitivity of growth inhibition by rhArg [11,13]. In contrast to ADIPEG20, the sensitivity to rhArg in hepatocellular carcinoma and melanoma is independent of ASS expression. Here, we studied the gene expression profile of OCT and ASS, and investigated the effects of rhArg in prostate cancer cells.

\section{Results and discussion}

\section{Expression of OCT and ASS}

Quantitative real-time PCR was performed in prostate cancer cells to detect mRNA expression of ASS, OCT, and glyceraldehyde 3-phosphate dehydrogenase (GAPDH). Data were processed and presented with cycle threshold (Ct) value of each quantitated expression as listed in Table 1. Housekeeping gene GAPDH was used as a reference gene for quantitative expression analysis. The $\mathrm{Ct}$ is defined as the number of cycles required for the fluorescent signal to cross the threshold level. $\mathrm{Ct}$ is a relative measure of the target mRNA in the PCR, and inversely proportional to the amount of target mRNA. Ct value of 40 or higher means no amplification due to absent gene expression. Abundant expression of ASS mRNA was detected in all three cell lines. Expression of OCT mRNA was absent in LNCaP, and minimally detected in DU-145 and $\mathrm{PC}-3$.

\section{Cell viability after arginine deprivation by rhArg}

We further determined cell viability after 72-h exposure to rhArg at $0,0.001,0.01,0.1$ and $0.5 \mathrm{U} / \mathrm{ml}$. All 3 prostate cancer cell lines were very sensitive to arginine depletion with half maximal inhibitory concentration $\left(\mathrm{IC}_{50}\right)$ of rhArg less than or equal to $0.02 \mathrm{U} / \mathrm{ml}$. The $\mathrm{IC}_{50}$ of rhArg in these 3 prostate cancer cell lines was similar to
Table 1 Cycle threshold $(\mathrm{Ct})$ of argininosuccinate synthetase (ASS), ornithine carbamoyl transferase (OCT), and glyceraldehyde 3-phosphate dehydrogenase (GAPDH) from quantitative real-time PCR in LNCaP, PC-3, and DU-145 cells

\begin{tabular}{cccc}
\hline & LNCaP & PC-3 & DU-145 \\
\hline ASS $C_{t}$ & 21.07 & 26.20 & 22.77 \\
OCT $C_{t}$ & 40.00 & 36.82 & 39.01 \\
GAPDH $C_{t}$ & 16.53 & 17.21 & 17.75 \\
\hline
\end{tabular}

the reported values in melanoma and hepatoma cell lines lacking OCT activity $[11,13]$. We further investigated the function of OCT by incubating cells with $0.1 \mathrm{U} / \mathrm{ml}$ of rhArg $\left(>\mathrm{IC}_{50}\right)$ in the absence and presence of increasing concentrations of ornithine for $72 \mathrm{~h}$. We expected ornithine could rescue rhArg-mediated cytotoxicity if OCT was expressed and functional. As shown in Figure 1, supplement of ornithine failed to rescue cytotoxicity from rhArg in all 3 cell lines. These results were in consistent with deficient OCT gene expression as demonstrated by quantitative real-time PCR.

\section{Signaling of mammalian target of rapamycin (mTOR)}

Autophagy is mediated by lysosomal degradation, and is an alternative process of cell death [21]. Autophagy is induced by inhibition of mTOR, which is a key sensor and regulator of growth signal and environmental stress [22]. Deprivation of arginine inhibits mTOR pathway and dephosphorylates downstream targets such as 4EBP1 in Chinese hamster ovary and human melanoma cells $[17,23]$. To elucidate the mTOR signaling following arginine depletion by rhArg, we investigated the phosphorylation pattern of 4E-BP1. Since our preliminary result showed citrulline could partially reverse the cytotoxicity from rhArg, cells were treated with either citrulline, rhArg, or both for $48 \mathrm{~h}$, followed by Western blot analysis (Figure 2). Decreased phosphorylation of 4E-BP1 in PC-3 and DU-145 was noted upon exposure to rhArg, irrespective of citrulline supplement. However, phosphorylation pattern of 4E-BP1 did not change in LNCaP. Though inhibition of mTOR by rhArg was noted in PC-3 and DU-145, the partial reversal of cytoxicity by citrulline might not be related to mTOR signaling since we did not observe any difference in phosphorylation pattern of $4 \mathrm{E}-\mathrm{BP} 1$ in the presence or absence of citrulline.

\section{Measurement of apoptosis}

Apoptosis was determined by DNA fragmentation using TUNEL (terminal deoxynucleotide transferase dUTP nick end labeling) assay after 36-h co-culture of prostate cancer cells with rhArg. Purple, green, pink, blue, and orange histograms indicate levels of DNA fragmentation 


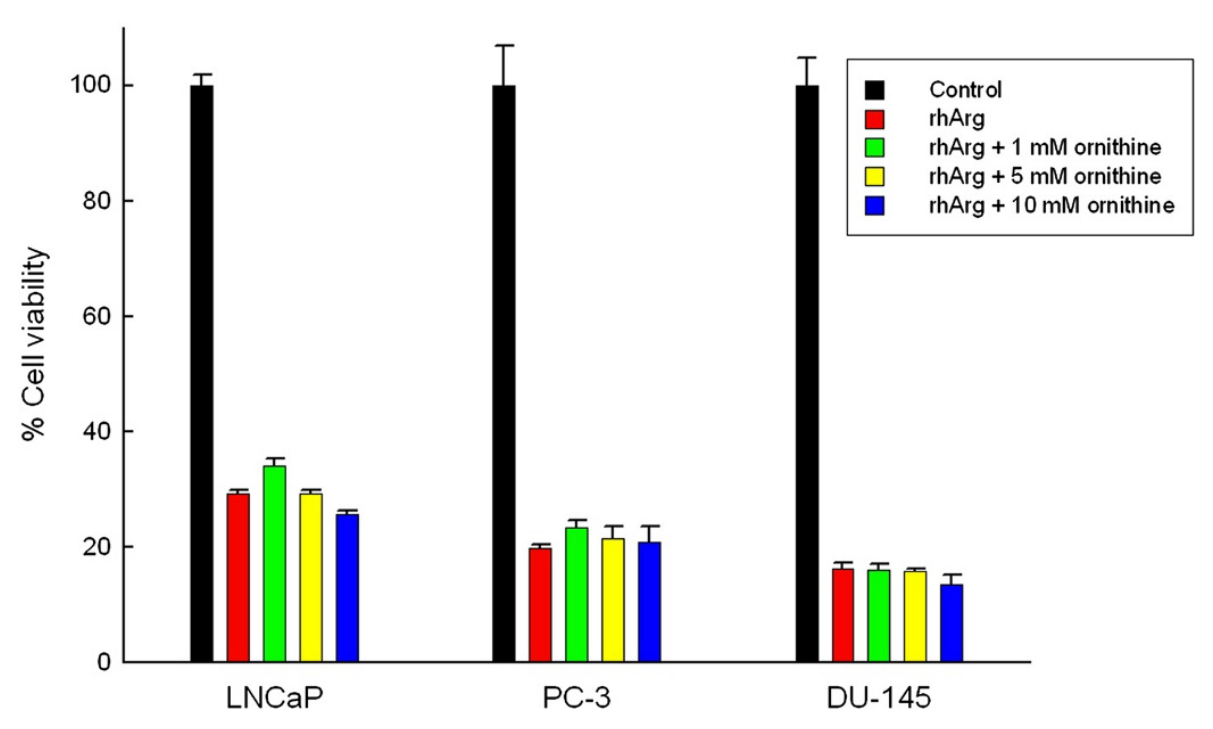

Figure 1 Cell viability after exposure to rhArg and various concentrations of orinithine. LNCaP, PC-3 and DU-145 cells were exposed to PBS (control), rhArg $(0.1 \mathrm{U} / \mathrm{ml})$, rhArg $(0.1 \mathrm{U} / \mathrm{ml})+1 \mathrm{mM}$ ornithine, rhArg $(0.1 \mathrm{U} / \mathrm{ml})+5 \mathrm{mM}$ ornithine, and $\mathrm{rhArg}(0.1 \mathrm{U} / \mathrm{ml})+10 \mathrm{mM}$ ornithine for $72 \mathrm{~h}$, then subjected to cell viability assay as mentioned in Methods. Values were reported as the average of 3 experiments with error bars showing standard error of the mean.

upon exposure with $0,0.001,0.01,0.1$, and $0.5 \mathrm{U} / \mathrm{ml}$ rhArg, respectively (Figure 3 ). The results demonstrated no induction of apoptosis after 36-h exposure of rhArg.

Deficiency in ASS expression renders cellular sensitivity against ADI-PEG20 in prostate cancer [18]. Both LNCaP and PC-3 have been shown to express ASS, and are resistant to arginine depletion by ADI-PEG20 [18]. In our study, all 3 prostate cancer cell lines including LNCaP and PC-3 expressed ASS but had either minimal or absent expression of OCT, and all 3 lines were highly susceptible to arginine deprivation by rhArg. Moreover, sensitivity to rhArg treatment was independent of hormone sensitivity and not affected by ASS expression in our study.

In human melanoma and prostate cancer cells with down-regulated ASS expression, treatment of ADI-PEG20 activates adenosine $5^{\prime}$-monophosphate-activated protein kinase (AMPK) due to decreased ATP levels upon arginine deprivation [18,24]. Activated AMPK further inhibits mTOR signaling by reducing phosphorylation of 4E-BP1, and leads to autophagy which is a cellular self-degrading process mediated by lysosomes. Kim et al. have shown arginine deprivation by ADI-PEG20 immediately activated AMPK, and formed intense autophagosome in CWR22Rv1 prostate cancer cells within 90 min of ADI-PEG20 exposure [18]. Onset of caspase-independent apoptosis in $~ 30 \%$ CWR22Rv1 cells did not occur until after 96-h exposure of ADI-PEG20. Similar findings of delayed-onset but caspasedependent apoptosis after arginine deprivation with 3 to 6 days exposure of either ADI-PEG20 or rhArg were reported by different groups [13,24].

Common stimuli can induce autophagy and apoptosis, which occur either in combined manner or sequential

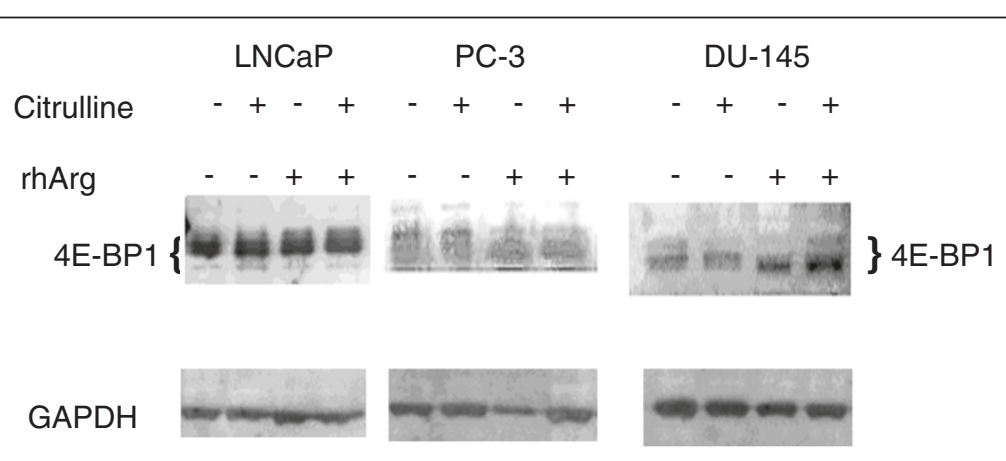

Figure 2 Western blot analysis of 4E-BP1 and GAPDH (as internal control) in the presence of citrulline, rhArg, or both for $48 \mathrm{~h}$. Hyperphosphorylated (slowly-migrating with higher molecular weight) and hypophosphorylated (fast-migrating) 4E-BP1 proteins were detected. 


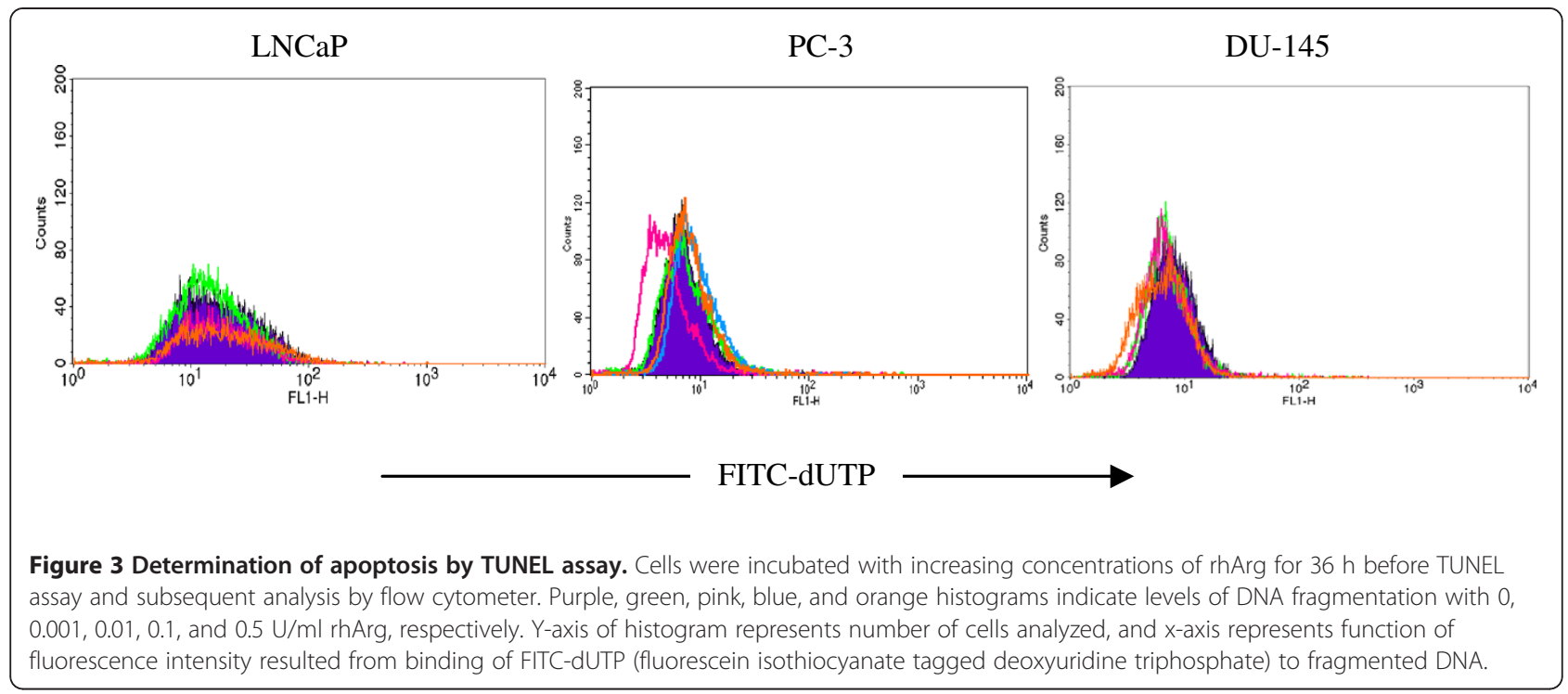

event [25]. It is unclear about the functional relationship between autophagy and apoptosis upon arginine deprivation with either ADI-PEG20 or rhArg. It is possible that upon initial arginine deprivation, autophagy is activated as a defense mechanism to suppress caspasedependent apoptosis. As arginine deprivation persists more than $72 \mathrm{~h}$, autophagy may give in to caspasedependent apoptosis in some cell types; whereas in certain cancer cells, autophagy lasting longer than $24 \mathrm{~h}$ may lead to caspase-independent form of programmed cell death (autophagic type II cell death) [26].

Using culture media deficient in L-arginine, Wheatley et al. studied the effects of arginine deprivation in 26 cancer cell lines, including PC-3 [27]. They demonstrated clear evidence of cell death during second day of arginine deprivation, and most of PC-3 cells died within 3 days. Furthermore, they observed significantly increased phagosome/ lysosome activity from 24 to $36 \mathrm{~h}$ after arginine deprivation, suggestive of lytic cell death such as autophagy rather than apoptosis. In this study, we did not identify any significant apoptosis induction after 36-h exposure of rhArg in all 3 cell lines. Additionally, inhibition of mTOR signaling manifested by decreased phosphorylation of 4E-BP1 was noted in DU-145 and PC-3 cells after 48-h exposure of rhArg. Our results are consistent with the report from Wheatley and others, indicating cell death by arginine deprivation in DU-145 and PC-3 is due to autophagic cell death.

Both rhArg and ADI are developed for arginine deprivation in cancer treatment, and currently undergoing clinical investigation. rhArg exhibits significant cytotoxicity against cancer cells such as prostate cancer, melanoma, and hepatocellular carcinoma with OCT deficiency. ADI is effective in tumor cells lacking ASS. Therefore, cancer can be ADI-resistant but rhArg-sensitive, and vice versa. Personalized medicine can be achieved by examining the expression of OCT and ASS in cancer specimen before subjecting cancer patients to arginine deprivation therapy.

\section{Conclusions}

rhArg causes significant cytotoxicity in LNCaP, DU-145 and $\mathrm{PC}-3$ prostate cancer cells. The cytotoxicity of rhArg correlates with deficient OCT gene expression, but is independent of hormone sensitivity and not affected by ASS gene expression. Inhibition of mTOR signaling, manifested by reduced phosphorylation of 4E-BP1, suggests autophagy is involved as alternative cell death mechanism. rhArg is a promising targeted agent for prostate cancer, and its activity and mechanism of action warrant further validation by clinical investigation.

\section{Methods}

\section{Cell culture}

DU-145, LNCaP and PC-3 human prostate cancer cells were obtained from the American Type Culture Collection (Manassas, VA). DU-145 and PC-3 are androgen-independent, and LNCaP is androgen-dependent [28]. Cell lines were maintained in RPMI 1640 medium (Life Technologies, Grand Island, NY) supplemented with $10 \%$ fetal bovine serum and antibiotics at $37^{\circ} \mathrm{C}$ in a humidified atmosphere of $5 \% \mathrm{CO}_{2}$. rhArg was kindly provided by BioCancer Treatments International Ltd. (Hong Kong, China), and was characterized as described previously [11].

\section{Quantitative real-time PCR}

Total RNA was extracted using TRIzol reagent (Life Technologies), and cDNA was transcribed from total RNA using SuperScript II RT kit (Life Technologies). Quantitative real-time PCR was performed in triplicate on a 7300 Real Time PCR System, using Gene 
Expression Assays for ASS, OCT, and GAPDH genes (Applied Biosystems, Foster City, CA). Data were processed and presented with $\mathrm{Ct}$ value of each gene expression.

\section{Cell viability assay}

Cells were plated at $10^{4}$ cells per well in a 96-well plate with increasing concentrations of rhArg at $0,0.001,0.01$, 0.1 and $0.5 \mathrm{U} / \mathrm{ml}$ for $72 \mathrm{~h}$ at $37^{\circ} \mathrm{C}$. Subsequently, cell viability was determined by a colorimetric method using CellTiter 96 Aqueous Non-radioactive Cell Proliferation Assay according to the manufacturer's protocol (Promega, Madison,WI).

\section{Protein extraction and Western blot analysis}

Protein extraction and Western blot analysis were carried out as previously described with some modifications [29]. After treatment, cells were washed twice with cold phosphate-buffered saline, and then resuspended in lysis buffer (phosphate-buffered saline containing 1\% Nonidet P-40, $0.5 \%$ sodium deoxycholate and $0.1 \%$ SDS) containing the protease inhibitors $(100 \mu \mathrm{g} / \mathrm{ml}$ phenylmethylsulfonyl fluoride, $25 \mu \mathrm{g} / \mathrm{ml}$ aprotinin, $25 \mu \mathrm{g} / \mathrm{ml}$ leupeptin, $10 \mu \mathrm{g} / \mathrm{ml}$ soybean trypsin inhibitor and $1 \mathrm{mM}$ sodium orthovanadate). The lysate was incubated on ice for $30 \mathrm{~min}$, passed through a 21 gauge needle twice, and then centrifuged at $15,000 \mathrm{x}$ g for $20 \mathrm{~min}$ at $4^{\circ} \mathrm{C}$. Protein concentration was determined using the Bio-Rad protein assay. Whole cell lysate containing $50 \mu \mathrm{g}$ of protein from each sample were used in immunoblotting, and subsequently the gels were electroblotted onto PVDF membranes (Immobilon-P, Millipore). Antibodies purchased from Cell Signaling Technology (Danvers, MA) were used to detect the proteins of interest. The horseradish peroxidase conjugated antibodies against mouse, rabbit and goat IgG were used as secondary antibodies (SigmaAldrich, St. Louis, MO). The secondary antibody binding was detected by ECL Plus chemiluminescent reagents and analyzed by Storm image analysis systems (GE Healthcare Biosciences, Piscataway, NJ).

\section{Apoptosis}

Apoptosis was determined by DNA fragmentation using ApoDirect TUNEL assay kit from Millipore (Billerica, MA) based on supplier's instruction. Briefly, $10^{6}$ Cells were incubated with increasing concentrations of rhArg for $36 \mathrm{~h}$. Afterwards, DNA breaks were fluorescently labeled with fluorescein isothiocyanate, and cells were analyzed by FACScan flow cytometer (Becton Dickinson Biosciences, San Jose, CA) using Cell Quest Pro software.

\section{Statistical analysis}

All experiments have been performed at least twice with similar results, and the results of one representative experiment are reported. Cell viability results are reported as the average of 3 experiments with error bars representing standard error of the mean as shown in Figure 1.

\section{Abbreviations}

OCT: Ornithine carbamoyl transferase; ASS: Argininosuccinate synthetase; ADI: Arginine deiminase; ADI-PEG20: Pegylated ADI; rhArg: Recombinant human arginase; GAPDH: Glyceraldehyde 3-phosphate dehydrogenase; Ct: Cycle threshold; $I_{50}$ : Half maximal inhibitory concentration; mTOR: Mammalian target of rapamycin; TUNEL: Terminal deoxynucleotide transferase dUTP nick end labeling; AMPK: Adenosine 5'-monophosphateactivated protein kinase.

\section{Competing interests}

PNMC is the chief executive officer of Bio-Cancer Treatments International Ltd, which manufactures rhArg. The rest of the authors declare no competing interest.

\section{Author details}

${ }^{1}$ Department of Surgery, Saint Louis University, St. Louis, MO, USA. ${ }^{2}$ Department of Applied Biology and Chemical Technology and Lo Ka Chung Centre for Natural Anti-Cancer Drug Development, The Hong Kong Polytechnic University, Hung Hom, Kowloon, Hong Kong, China. ${ }^{3}$ Bio-Cancer Treatments International Ltd., Hong Kong Science Park, Bio-informatics Building, Hong Kong, China. ${ }^{4}$ Division of Medical Oncology and Hematology, Loma Linda University, Loma Linda, CA92354, USA.

\section{Authors' contributions}

ECH, WHL, YCL, PNMC and CTH designed the experiments. ECH and SMK performed the experiments. ECH and CTH wrote the paper. All authors read and approved the final manuscript.

Received: 28 March 2012 Accepted: 30 April 2012 Published: 30 April 2012

\section{References}

1. Lind DS: Arginine and cancer. J Nutr 2004, 134(10 Suppl):2837S-2841S. discussion 2853S.

2. Morris SM Jr: Arginine metabolism: boundaries of our knowledge. J Nutr 2007, 137(6 Suppl 2):1602S-1609S.

3. Raijman L: Citrulline synthesis in rat tissues and liver content of carbamoyl phosphate and ornithine. Biochem J 1974, 138(2):225-232.

4. Delers A, Szpirer J, Szpirer C, Saggioro D: Spontaneous and 5-azacytidineinduced reexpression of ornithine carbamoyl transferase in hepatoma cells. Mol Cell Biol 1984, 4(4):809-812.

5. Bach SJ, Swaine D: The effect of arginase on the retardation of tumour growth. Br J Cancer 1965, 19:379-386.

6. Wheatley DN: Arginine deprivation and metabolomics: important aspects of intermediary metabolism in relation to the differential sensitivity of normal and tumour cells. Semin Cancer Biol 2005, 15(4):247-253.

7. Tytell AA, Neuman RE: Growth response of stable and primary cell cultures to I-ornithine, I-citrulline, and I-arginine. Exp Cell Res 1960, 20 (1):84-91.

8. Kraemer PM: Interaction of mycoplasma (Pplo) and murine lymphoma cell cultures: prevention of cell lysis by arginine. Proc Soc Exp Biol Med 1964, 115:206-212.

9. Dillon BJ, Prieto VG, Curley SA, Ensor CM, Holtsberg FW, Bomalaski JS, Clark MA: Incidence and distribution of argininosuccinate synthetase deficiency in human cancers: a method for identifying cancers sensitive to arginine deprivation. Cancer 2004, 100(4):826-833.

10. Ensor CM, Holtsberg FW, Bomalaski JS, Clark MA: Pegylated arginine deiminase (ADI-SS PEG20,000 mw) inhibits human melanomas and hepatocellular carcinomas in vitro and in vivo. Cancer Res 2002, 62 (19):5443-5450.

11. Cheng PN, Lam TL, Lam WM, Tsui SM, Cheng AW, Lo WH, Leung YC: Pegylated recombinant human arginase (rhArg-peg5,000 mw) inhibits the in vitro and in vivo proliferation of human hepatocellular carcinoma through arginine depletion. Cancer Res 2007, 67(1):309-317. 
12. Lam TL, Wong GK, Chong HC, Cheng PN, Choi SC, Chow TL, Kwok SY, Poon RT, Wheatley DN, Lo WH, et al: Recombinant human arginase inhibits proliferation of human hepatocellular carcinoma by inducing cell cycle arrest. Cancer Lett 2009, 277(1):91-100.

13. Lam TL, Wong GK, Chow HY, Chong HC, Chow TL, Kwok SY, Cheng PN, Wheatley DN, Lo WH, Leung YC: Recombinant human arginase inhibits the in vitro and in vivo proliferation of human melanoma by inducing cell cycle arrest and apoptosis. Pigment Cell Melanoma Res 2010, 24(2):366-376.

14. Miyazaki K, Takaku H, Umeda M, Fujita T, Huang WD, Kimura T, Yamashita J, Horio T: Potent growth inhibition of human tumor cells in culture by arginine deiminase purified from a culture medium of a Mycoplasmainfected cell line. Cancer Res 1990, 50(15):4522-4527.

15. Izzo F, Marra P, Beneduce G, Castello G, Vallone P, De Rosa V, Cremona F, Ensor CM, Holtsberg FW, Bomalaski JS, et al: Pegylated arginine deiminase treatment of patients with unresectable hepatocellular carcinoma: results from phase I/II studies. J Clin Oncol 2004, 22(10):1815-1822.

16. Ascierto PA, Scala S, Castello G, Daponte A, Simeone E, Ottaiano A, Beneduce G, De Rosa V, Izzo F, Melucci MT, et al: Pegylated arginine deiminase treatment of patients with metastatic melanoma: results from phase I and II studies. J Clin Oncol 2005, 23(30):7660-7668.

17. Feun L, You M, Wu CJ, Kuo MT, Wangpaichitr M, Spector S, Savaraj N: Arginine deprivation as a targeted therapy for cancer. Curr Pharm Des 2008, 14(11):1049-1057.

18. Kim RH, Coates JM, Bowles TL, McNerney GP, Sutcliffe J, Jung JU, GandourEdwards R, Chuang FY, Bold RJ, Kung HJ: Arginine deiminase as a novel therapy for prostate cancer induces autophagy and caspaseindependent apoptosis. Cancer Res 2009, 69(2):700-708.

19. Bach SJ, Hawkins RA, Swaine D: A short method for the purification of arginase from ox liver. Biochem J 1963, 89:263-265.

20. Tsui SM, Lam WM, Lam TL, Chong HC, So PK, Kwok SY, Arnold S, Cheng PN, Wheatley DN, Lo WH, et al: Pegylated derivatives of recombinant human arginase (rhArg1) for sustained in vivo activity in cancer therapy: preparation, characterization and analysis of their pharmacodynamics in vivo and in vitro and action upon hepatocellular carcinoma cell (HCC). Cancer Cell Int 2009, 9:9.

21. Hotchkiss RS, Strasser A, McDunn JE, Swanson PE: Cell death. N Engl J Med 2009, 361(16):1570-1583.

22. Jung $\mathrm{CH}$, Ro $\mathrm{SH}$, Cao J, Otto NM, Kim DH: mTOR regulation of autophagy. FEBS Lett 2010, 584(7):1287-1295.

23. Hara K, Yonezawa K, Weng QP, Kozlowski MT, Belham C, Avruch J: Amino acid sufficiency and mTOR regulate p70 S6 kinase and elF-4E BP1 through a common effector mechanism. J Biol Chem 1998, 273(23); 14484-14494.

24. Savaraj N, You M, Wu C, Wangpaichitr M, Kuo MT, Feun LG: Arginine deprivation, autophagy, apoptosis (AAA) for the treatment of melanoma. Curr Mol Med 2010, 10(4):405-412.

25. Maiuri MC, Zalckvar E, Kimchi A, Kroemer G: Self-eating and self-killing: crosstalk between autophagy and apoptosis. Nat Rev Mol Cell Biol 2007, 8 (9):741-752.

26. Gozuacik D, Kimchi A: Autophagy as a cell death and tumor suppressor mechanism. Oncogene 2004, 23(16):2891-2906.

27. Scott L, Lamb J, Smith S, Wheatley DN: Single amino acid (arginine) deprivation: rapid and selective death of cultured transformed and malignant cells. Br J Cancer 2000, 83(6):800-810

28. van Bokhoven A, Varella-Garcia M, Korch C, Johannes WU, Smith EE, Miller HL, Nordeen SK, Miller GJ, Lucia MS: Molecular characterization of human prostate carcinoma cell lines. Prostate 2003, 57(3):205-225.

29. Hsueh CT, Wu YC, Schwartz GK: UCN-01 suppresses E2F-1 mediated by ubiquitin-proteasome-dependent degradation. Clin Cancer Res 2001, 7 (3):669-674.

\section{Submit your next manuscript to BioMed Central and take full advantage of:}

- Convenient online submission

- Thorough peer review

- No space constraints or color figure charges

- Immediate publication on acceptance

- Inclusion in PubMed, CAS, Scopus and Google Scholar

- Research which is freely available for redistribution

Submit your manuscript at www.biomedcentral.com/submit
Ciomed Central 